Georgia State University

ScholarWorks @ Georgia State University

\title{
Age and Body Satisfaction Predict Diet Adherence in Adolescents with Inflammatory Bowel Disease
}

\author{
Christina H. Vlahou \\ Lindsey L. Cohen \\ Georgia State University, Ilcohen@gsu.edu \\ Amanda M. Woods \\ awoods37@gmail.com \\ Jeffrey D. Lewis \\ Benjamin D. Gold
}

Follow this and additional works at: https://scholarworks.gsu.edu/psych_facpub

Part of the Psychology Commons

\section{Recommended Citation}

Vlahou, C. V., Cohen, L. L., Woods, A., Gold, B., \& Lewis, J. (2008). Age and body satisfaction predict diet adherence in adolescents with inflammatory bowel disease. Journal of Clinical Psychology in Medical Settings, 15(4), 278-286. DOI: 10.1007/s10880-008-9125-8

This Article is brought to you for free and open access by the Department of Psychology at ScholarWorks @ Georgia State University. It has been accepted for inclusion in Psychology Faculty Publications by an authorized administrator of ScholarWorks @ Georgia State University. For more information, please contact scholarworks@gsu.edu. 


\section{Running head: PREDICTORS OF IBD ADHERENCE}

Age and Body Satisfaction Predict Diet Adherence in Adolescents with Inflammatory Bowel

Disease

$$
\begin{aligned}
& \text { Christina H. Vlahou } \\
& \text { Lindsey L. Cohen } \\
&
\end{aligned}
$$

${ }^{1}$ Department of Psychiatry and Health Behavior, Medical College of Georgia, Augusta, Georgia ${ }^{2}$ Department of Psychology, Georgia State University, Atlanta, Georgia

${ }^{3}$ Children's Center for Digestive Health Care, LLC., Atlanta, Georgia

${ }^{4}$ Division of Gastroenterology, Emory Children's Center; Emory University School of Medicine, Atlanta, Georgia

Correspondence concerning this article should be addressed to:

Dr. Lindsey L. Cohen

Department of Psychology,

Georgia State University, Atlanta, Georgia 30302-5010

Office Number: 404-413-6263

Fax: 404-413-6207

Email: 1lcohen@gsu.edu 


\begin{abstract}
The aim of the current study was to determine whether age and body satisfaction predict dietary adherence in adolescents with Inflammatory Bowel Disease (IBD), and whether older females are less adherent than younger males and females. Forty-four participants aged 10-21 with IBD were recruited. Participants provided informed consent and demographics. Body satisfaction was measured by a questionnaire and a task in which participants selected their current and ideal body image out of silhouettes depicting bodies ranging from underweight to obese. Adherence was measured by marking a 100mm visual analog scale, the 1-week completion of a dietary $\log$, and a questionnaire evaluating coping strategies used for overcoming obstacles to dietary adherence. Age was related to dietary adherence, with younger children being more likely to adhere. Participants more satisfied with their body reported better dietary adherence. Findings remained consistent across multiple measures of body satisfaction and adherence. Healthcare providers and parents should be informed of these findings in order to improve adherence.
\end{abstract}

Key Words: Inflammatory Bowel Disease; Body Satisfaction; Diet Adherence; Pediatric Psychology 
Age and Body Satisfaction Predict Diet Adherence in Adolescents with Inflammatory Bowel Disease

Inflammatory bowel disease (IBD) is a chronic, incurable disorder where the intestinal system becomes inflamed, resulting in a host of complications such as abdominal pain and diarrhea. The inflammation and scar tissue causes temporary and permanent damages to the intestines resulting in poor absorption of nutrients from food, leading to malnutrition and delayed growth. A number of patients with IBD have increased nutritional and caloric needs due to delayed growth or surgery, and/or have low tolerance for solid foods due to the severity of their illness, in which case, dietary restrictions, nutritional supplements, elemental diet, or total parenteral nutrition (TPN) might be recommended or required (CCFA, 2005).

Patients with IBD are often encouraged to avoid particular foods that can cause aggravation, dyspepsia or indigestion, or can result in intestinal obstructions (MacDermott, 2007). Further, oral intake might be adjusted related to abdominal pain or "flare-ups." Thus, dietary recommendations contribute to the treatment regimen of IBD (Booth, 1991; Powers, 1997; Woolner et al., 1998). Research examining diet adherence in children with IBD (Booth, 1991; Woolner et al., 1998) and other pediatric chronic illness (Mackner, McGrath, \& Stark, 2001; Stark, 2000), suggests that it is poor. It is important to note that there are no general recommendations that apply to all patients with IBD; patients usually resort to a combination of physician/dietician recommendations and personal trial and error in order to identify diet guidelines appropriate for them.

A critical variable to consider when examining adherence in chronic illness is the developmental level of the patients (La Greca \& Bearman, 2003; Manne, 1998; Rapoff, 1999). In IBD, most individuals are diagnosed between the ages of 15 and 35, and estimations of those 
affected under the age of 18 range from $10 \%$ of all patients diagnosed (CCFA, 2005) to $30 \%$ (Escher \& Taminiau, 2001). Unfortunately, research across a number of pediatric chronic illnesses suggests that adolescents are less adherent than younger children (for a review, see La Greca \& Bearman, 2003; Manne, 1998; Rapoff, 1999). In fact, there are data suggesting that dietary adherence is particularly poor in adolescent patients with IBD (Booth, 1991).

Given that both IBD and treatment for IBD (e.g., corticosteroids, diet recommendations) can impact a patients' body size, weight, and shape, body satisfaction is a central concern with this population (Dunker et al., 1998; Casati et al., 2000; De Rooy et al., 2001). Body satisfaction or body image can be defined as both the personal perception of one's body and the cognitions and emotions associated with one's body, in addition to the degree to which one is satisfied with one's physical appearance (Kelsay, Hazel, \& Wamboldt, 2005). No research to date has examined whether body satisfaction impacts IBD diet adherence, although body satisfaction has been found to adversely impact adherence in other chronic illness populations (Abbott et al., 2000; Joyce, 2002; Walters, 2001). However, it is important to note that an association between poor body image and poor adherence might not be present for both genders as it is well established that eating disorders are more prevalent in females in mid to late adolescence (DSMIV-TR, 2000). Furthermore, body dissatisfaction manifests differently in males and females such that girls tend to focus on a lower overall body weight whereas boys tend to desire an increase in muscle mass in concordance with low body fat (Pope, Phillips, \& Olivardia, 2000). Also, females are reported to experience a greater increase in body dissatisfaction secondary to medication and dietary regimens than males, even when they experience less weight gain than males (Antisdel \& Chrisler, 2000). 
In the current study of IBD patients, based on literature from IBD and other chronic illness diagnoses, it was hypothesized that 1) older adolescents would be less adherent than younger pre-adolescent children, and that 2) body satisfaction would relate to adherence, with adolescents who were more satisfied with their body being more adherent. It was expected that body satisfaction would influence adherence above and beyond age. However, the relation between body satisfaction and adherence was expected to hold true only for female participants, who are socialized to desire smaller, thinner bodies. Given that older adolescents are more likely to have body satisfaction concerns than the younger pre-adolescents, we predicted a 3-way interaction between age, gender, and body satisfaction in predicting diet adherence.

Method

\section{Participants}

Fifty-seven participants with a diagnosis of IBD (i.e., Crohn's disease or ulcerative colitis) were recruited for the current study. Of the 57 recruited, 44 (77.2\%) participants (22 males, 22 females) endorsed following self or physician - imposed dietary guidelines due to their illness and were included in the study, although the entire sample endorsed having had to adhere to a dietary regimen at some point during the course of their illness. Their ages ranged from 10 to 21 years $(M=16.6$ years, $S D=2.4$ years $)$. For the sake of parsimony, children, adolescents, and young adults are all termed "adolescents" in the current study. Thirty-seven participants were identified as Caucasian (84.1\%), $6(13.6 \%)$ as Black, and $1(2.3 \%)$ as multiracial. The mean Body Mass Index $(\mathrm{BMI})$ was $22.7(S D=4.88)$ for males, and $23.73(S D=4.16)$ for females, within average ranges according to the United States BMI population norms for girls and boys aged 2 to 20 years old (National Center for Health Statistics \& National Center for Chronic 
Disease Prevention and Health Promotion., 2000). Their ages at the time of diagnosis ranged from 5 to 18 years old $(M=12.38$ years, $S D=3.04$ years $)$.

\section{Measures}

Family Information Form. A family information form was administered to obtain general background data such as age, gender, age of diagnosis, disease history, and other relevant variables. These data were used for descriptive purposes and age was examined as a predictor of diet adherence.

Body Satisfaction Questionnaire (BSQ). To assess body satisfaction a modified version of an 8-item questionnaire ("Body Image Questionnaire") used in a previous study by Dunker and colleagues (1998) who examined body image differences among individuals with IBD postsurgically was administered and yielded two factors: a body image factor and a cosmetic factor. The body image subscale was reported to measure the patients' perceptions of, and satisfaction with, their own bodies and their attitudes toward their bodily appearance. The cosmetic factor was reported to assess the degree of the patients' satisfaction with the physical appearance of their surgical scar. Internal consistency for this measure has been shown to be good for the body image (Cronbach's alpha $=.80)$ and cosmetic (Cronbach's alpha $=.83$ ) scales (Dunker et al., 1998).

For the purpose of this study, 6 of the items were modified to fit the current sample and only a body image factor score was derived, given that there was no component related to surgery in the present study. The modifications that took place were changing "the operation" to "the diagnosis" or "the disease" (e.g., Are you less satisfied with your body since the operation?" became "Are you less satisfied with your body since your diagnosis?") and "scar" to "body" (e.g. "How satisfied are you with your scar" was changed to "How satisfied are you with your body"). 
The modified questionnaire contained a total of 9 questions. Six questions asked about body satisfaction in direct relation to the illness (e.g., "Do you think the disease has damaged your body on the outside?" or "Do you feel less attractive as a result of your disease or treatment?") and were answered on a four-point Likert scale ranging from "No, not at all" to "Yes, extremely." Three questions asked the respondents to provide general satisfaction ratings for their body (e.g., “Overall, how self-confident do you feel after the disease?”) and were answered on a seven-point Likert scale ranging from "Very unsatisfied/Not at all confident" to "Very satisfied/Very confident." The total questionnaire score was computed and a higher numerical score was indicative of more body satisfaction. Internal consistency within the current total sample was good (alpha $=.85)$. It was more reliable for girls $($ alpha $=.86)$ than for boys $($ alpha $=.76)$.

Body Image Assessment (BIA). The BIA was utilized as an additional measure of body satisfaction. The BIA procedure was originally developed for adults by Williamson et al. (1989). However, a preadolescent version (BIA-P; Veron-Guidry \& Williamson, 1996) was later developed and was used in the current study as the age range of the current sample was mainly within the pre-adolescent to adolescent range (i.e., 10 to 21 years old). It is important to note that the latter study (Veron-Guidry \& Williamson, 1996) on this measure aimed to develop norms and evaluate the validity and reliability for the original adult version for younger age groups; the pre-adolescent silhouettes depict vague bodily figures with adequate secondary sex characteristics and were thus considered appropriate for use with adolescents and young adults.

The BIA-P measure includes four sets of body image silhouettes, mounted on separate 6 in. by 9 in. cards corresponding to male and female preadolescents. Each set of silhouettes has nine body sizes ranging from very thin to obese. Administration was in accordance with the 
guidelines provided by Veron-Guidry and Williamson (1996) in which participants were asked to identify their current body size (CBS) and ideal body size (IBS). A body dysphoria score (BDS) was derived from the difference between the two (i.e., BDS = IBS - CBS). The higher the discrepancy between current and ideal body types, the higher the BDS.

Veron-Guidry \& Williamson (1996) reported a test-retest reliability of this measure of .94 for the CBS and .93 for the IBS. A one-week reliability check yielded a Pearson $r$ of .79 ( $p$ $<.01)$ and $.67(p<.01)$ for the CBS and IBS respectively. Concurrent validity was demonstrated by the significant relation $(r=.33, p<.01)$ between the BIA discrepancy score (CBS-IBS) and a measure of eating disorder symptoms. Concurrent validity is suggested by the significant correlation between the body dysphoria score (BDS) and the Body Satisfaction Questionnaire (BSQ), $r=-.42, \mathrm{p}<.01$.

Situational Obstacles to Dietary Adherence (SODA). The SODA (Schlundt et al., 1996) was used as a proxy for dietary adherence, one of the dependent variables in the current study. Although it is not a direct measure of dietary adherence, it was selected in order to capture another dimension of the multi-faceted construct of adherence. The SODA was initially developed for the purpose of evaluating the ability of adolescents with insulin-dependent diabetes mellitus (IDDM) to cope with situational obstacles to their dietary adherence (Schlundt et al., 1996). The original measure was comprised of two scales, a confidence scale, indicating how confident the adolescent feels that he or she could overcome a variety of challenges to dietary adherence, and a behavioral scale measuring use of behavioral strategies to overcome such challenges. Respondents were required to indicate how confident they were that they could solve each problem (confidence scale), and then rate how frequently they were likely to use each of five possible strategies for overcoming this obstacle. All answers were provided on a Likert 
scale ranging from one ("Not at all confident" or "Never do") to seven ("Completely confident" or "Always do"). Schlundt et al. (1996) reported a Cronbach Alpha of .93 for internal consistency of the confidence scale and .62 for internal consistency across subscales. For the purpose of determining validity, Schlundt et al. (1996) compared the confidence scale to another scale measuring self-efficacy in diabetes and yielded a Cronbach Alpha of .83.

For the purposes of the current study, the instructions, as well as some of the vignettes and the potential strategies, were either modified from the original version so that they apply to IBD rather than IDDM, or completely excluded if not applicable to IBD. Given the extensive length of the original measure, the current study utilized an abbreviated version by removing duplicate vignettes that shared themes and content. The vignettes were evaluated by two separate raters and rated for their degree of similarity. For any two vignettes that both raters considered similar, one was included and the other excluded, resulting in 18 vignettes used in the current study.

A total score for the behavioral scale was computed by summing up all behavioral scale items, and was the score used in the current analyses. Reverse coding was applied where necessary so that a higher total score reflected better overall ability to cope with dietary adherence obstacles. A total score for the confidence scale was computed by summing up all confidence scale items and was used for descriptive analyses. Within the current sample, internal consistency was excellent, confidence scale $($ alpha $=.92)$ and behavioral scale (alpha $=.94)$.

Diet Adherence Perception. Adolescents were asked to self-report their personal perception of their diet adherence ("Overall, how is your adherence to your diet? Do you eat according to medical recommendations?") as measured by making a vertical mark on a 
horizontal 100mm line ranging from "Never" to "Always". This question was used as a global measure of diet adherences.

Diet Adherence Log. As a measure of diet adherence, participants were asked to record their perception of dietary adherence daily for a period of 7 days. This is based on research suggesting that patient perception of adherence might be a better measure than actual adherence, and that the shorter the recall periods and the more detailed and objective the questions asked, the more accurate the self-reports (La Greca \& Bearman, 2003). Participants were provided with logs that resembled a one-week calendar and asked to complete them at the end of each day by recording their perceived adherence to dietary restrictions/recommendations, on a three-point scale (1, "I only ate good foods," 2, "I ate one thing I shouldn't eat," 3, "I ate more than one thing I shouldn't eat”). Participants were also provided with a stamped, self-addressed envelope and asked to mail the logs to the researcher in one week, once completed.

Participants reported whether they adhered to their diet completely, deviated a little, or deviated a lot and received a corresponding score of 1, 2, or 3 on each day of the week, with lower scores equaling more adherent behavor. Scores were then reverse-coded, so that a higher score was indicative of more dietary adherence. The total score for diet adherence was used as the dependent variable in this study. The diet adherence log total score was found to correlate with the SODA adherence score, $r=45, p<.01$, which suggests good concurrent validity for the measures.

\section{Procedure}

This study received the approval of the Georgia State University Institutional Review Board (IRB), the Emory University IRB, and the Children's Healthcare of Atlanta IRB. There were two methods of recruitment. First, participants were recruited in person at two sites, the 
Emory Children's Center (ECC) and the Children's Center for Digestive Healthcare (CCDHC). Recruitment was conducted somewhat differently at the two sites due to different site policies. At ECC, parents and their children $(n=16)$ were approached directly by the examiner during their wait for their physician visit or their Remicade ${ }^{\circledR}$ infusion, presented with the study and asked to participate; whereas, at CCDHC, a staff member provided the children and their families $(n=37)$ with a recruitment letter during their Remicade ${ }^{\circledR}$ infusion, and only introduced them to the researcher if they were interested in participating. Second, a letter was sent out by the Crohn's and Colitis Foundation of America (CCFA) addressed to the parents of the children who participate in a summer camp for children with Crohn's disease and ulcerative colitis. The letter described the current study and requested that the parents call the researcher to volunteer for their child to participate. For those parents who responded $(n=4)$, a day was arranged for the researcher to meet with the parent and adolescent at a location of their choice, which in all cases was their home.

In all cases, adolescents with IBD and their parents were informed that they were being invited to participate in a study aiming to assist individuals with IBD in taking their medication and sticking to their diet regimens. To protect privacy and prevent external threats to validity such as biased responding, all measures were administered in the form of a self-report questionnaire. For the BIA, which required that the participants physically point to the silhouette of their choice, an effort was made to administer this brief measure in a separate, private room. All interested families completed parent consent and child assent forms, as well as HIPAA designated forms providing authorization to release personal health information for research purposes. The demographic form was initially completed by the adolescent with the assistance of the parent, unless the adolescent was older than 18 and/or the parent was not present. The SODA, 
BIQ, and BIA were then administered to the participant in random order. Finally, the participant was provided with the diet adherence log sheet and instructions on how to complete it. Before leaving, the researcher thanked the families for participation and provided them with a compensation fee of $\$ 10$.

Results

\section{Preliminary Analyses}

The three data collection sites were compared to evaluate whether they were equivalent. Specifically, analyses of variance (ANOVAs) for interval and ratio data and chi-square tests for all nominal data were performed on all demographic and study measures to compare scores obtained across sites. No differences were found and therefore subsequent analyses were conducted on the entire sample collapsed across data collection sites. Of the 44 participants enrolled in the study with dietary restrictions, $91 \%$ returned complete diet logs. The large majority of the adolescents $(89 \%)$ reported that their adherence during the week of the study was equivalent to that of other weeks. Descriptive statistics were computed for both of the predictor measures (age, body satisfaction) and the dependent variable (SODA, dietary adherence log, dietary adherence perception). These data are summarized in Table 1. For measures of body satisfaction, an ANOVA was run to compare males and females. As predicted, females reported significantly less body satisfaction than males on both the BSQ $(F=5.56, p<.05)$ and the BIA $(F=6.12, p<.05)$.

Regression Assumptions. A series of regression diagnostic tests were performed to ensure that the data met the assumptions of regression. Normality was explored by plotting a histogram and examining skewness statistics. Linearity was examined by plotting a scattergram, and homoscedasticity was explored by plotting the standardized predicted residuals for each 
predictor variable. The results of this analysis revealed that the assumptions of regression were met and no transformations were needed.

\section{Primary Analyses}

Two sets of three hierarchical linear regression analyses (total of six) were conducted, one set for each of the two measures of body satisfaction (i.e., BIA and BSQ) predicting each of the three measures of diet adherence (i.e., SODA, self-report, and diet log) (Table 2). Prior to running the analyses and creating the interaction terms, age, gender, and body satisfaction (BIA and BSQ) were centered according to recommendations by Aiken and West (1991). The regressions consisted of four steps. Age was entered in the first step, followed by body satisfaction (BSQ or BIA) in the next step. The two-way body satisfaction (BSQ or BIA) by gender interaction was entered into the third step, and the three-way age by body satisfaction (BSQ or BIA) by gender interaction was entered into the fourth step. The main effects and interaction terms were not interpreted unless the amount of variance accounted for within the

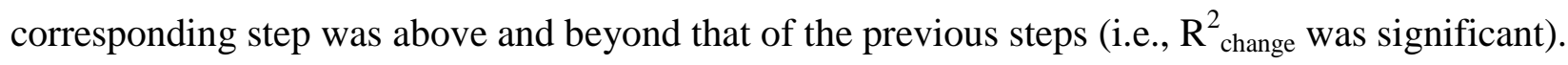

First, analyses were conducted using the SODA as the outcome variable. Results of this regression analysis revealed a significant main effect of age $(\beta=-.42, p<.01)$ on dietary adherence (SODA), accounting for approximately $17 \%$ of the variance $\left(\mathrm{R}_{\text {change }}^{2}=.17 ; \mathrm{F}_{\text {change }}(1\right.$, $41)=8.57, p=.006)$. This finding suggests that as hypothesized, older adolescents were less likely to adhere to their dietary regimen. Neither of the two-way or three-way interactions were found to be significant. Using the BSQ for body satisfaction, a main effect was found $(\beta=.36, p$ $<.05)$, which accounted for $12 \%$ of the variance in diet adherence (SODA) $\left(\mathrm{R}_{\text {change }}^{2}=.12 ; \mathrm{F}_{\text {change }}\right.$ $(2,39)=3.43, p=.042)$. Body satisfaction as measured by the BIA yielded a nearly significant main effect on dietary adherence (SODA) $(\beta=-.33, p<.1)$ accounting for $10 \%$ of the variance 
above and beyond baseline levels of age $\left(\mathrm{R}_{\text {change }}^{2}=.10 ; \mathrm{F}_{\text {change }}(2,39)=2.81, p=.072\right)$. It has been suggested that effect sizes may be used as a measure of significance rather than conventional $p$ value cutoffs (Kraemer et al., 1999); given the small sample size and the effect size of body satisfaction, this finding may be considered significant and suggests that as hypothesized, more body dissatisfaction was related to less dietary adherence. When the BSQ was entered into the third step of a separate analysis after age and the BIA, it was found to account for $1 \%$ of the variance in predicting diet adherence above and beyond age and the BIA; although it approached, it did not reach significance $\left(\mathrm{R}_{\text {change }}^{2}=.01 ; \mathrm{F}_{\text {change }}(2,38)=2.55, p\right.$ $=.091)$.

Another analysis was conducted substituting diet logs as a measure of dietary adherence. A main effect of body satisfaction was found for both the BSQ $(\beta=.19, p<.01)$ and the BIA $(\beta$ $=-.33, p<.01)$ with participants who were more satisfied with their body reporting more dietary adherence $\left(\mathrm{BSQ}: \mathrm{R}_{\text {change }}^{2}=.23 ; \mathrm{F}_{\text {change }}(2,36)=5.34, p=.009 ; \mathrm{BIA}: \mathrm{R}_{\text {change }}^{2}=.28 ; \mathrm{F}_{\text {change }}(2,36)\right.$ $=7.2, p=.002)$. Finally, using the Diet Adherence Perception score as the dependent variable, both age $\left(\mathrm{R}_{\text {change }}^{2}=.07 ; \mathrm{F}_{\text {change }}(1,53)=3.77, p=.06\right)$ and BIA body satisfaction $\left(\mathrm{R}_{\text {change }}^{2}=.10\right.$; $\left.\mathrm{F}_{\text {change }}(2,51)=3.1, p=.06\right)$ approached significance, suggesting that younger children and participants who were more satisfied with their body perceived themselves as more adherent.

Although the body satisfaction by gender interaction was not found to be significant, when regressions were run separately for male and females, the effect of body satisfaction on dietary adherence remained significant only for the female participants (BIA: SODA: $R^{2}=.40 ; F$ $(1,19)=6.47, p=.001$; Diet Log: $R^{2}=.22 ; F(1,16)=5.59, p=.03$; Diet Adherence Perception: $\left.R^{2}=.21 ; F(1,22)=6.96, p=.02 ; \mathrm{BSQ}: \mathrm{SODA}: R^{2}=.22 ; F(1,19)=6.71, p=.02\right)$. Genders were compared using a one-way analysis of variance (ANOVA) on diet log and SODA; no 
differences were found for the SODA $(F=.03, p=.86)$, but females reported less adherence than males on the diet $\log (F=9.22, p<.01)$. When females were compared to males on their confidence in their ability to overcome obstacles to dietary adherence as reported on the SODA, they were found to be significantly less confident $(F=4.29, p<.05)$. No correlation between confidence and age was found $(r=-.046, p=.77)$.

\section{Discussion}

This study is one of the first to examine diet adherence in a pediatric population with IBD. Many of our hypotheses were largely confirmed. First, age was found to be significantly related to self-reported adherence; as hypothesized, older adolescents reported being less likely to adhere to their diet on the one-week diet log. In addition, older children reported less ability to overcome obstacles to their dietary adherence. One possible interpretation of this finding is that even though children seek out more independence as they grow into adolescence, they may not simultaneously become more responsible or monitor their diet as would be ideal. Parental expectations and less parental monitoring might play a role as well. Competing factors might also come into play when children reach adolescence, such as need for peer recognition, romantic emotions, puberty, and increased academic pressure and demands. It is important to note however, that this finding could also be the result of older participants having higher standards and more self-demands in regard to their diet, or a better ability to accurately evaluate and report their dietary adherence compared to younger children. Older children are also more likely to have had experience with the disease for a longer period of time, and longer illness duration is one of the factors that have been associated with poorer adherence (for a review see Kane, 2006; López-Sanromán, \& Bermejo, 2006). Alternatively, younger children might be more prone to providing socially desirable responses than adolescents. 
Second, body satisfaction was found to predict diet adherence above and beyond age. As hypothesized, participants who were less satisfied with their body were less likely to follow their diet regimen. This finding suggests that body satisfaction is related to patients' likelihood to choose foods that are healthy and compatible with their chronic illness. On the other hand, this finding might indicate that participants who are less likely to adhere to their diet are also less likely to be satisfied with their bodily appearance. Other factors could also be affecting this relation, such as an overall negative response style.

The differences across gender deserve discussion. The result that female patients with IBD are less satisfied with their bodies than male patients with IBD parallels findings of gender differences in body satisfaction in the general population (e.g., Lokken et al., 2003; Pharres, Steinburg, \& Thompson, 2004). Although we did not find a body satisfaction by gender interaction, when the analyses were run separately for each gender, a significant effect of body dissatisfaction on dietary adherence was found for females but not for males. Healthcare providers may consider discussing this factor in relation to diet regimens, as body satisfaction could potentially influence both boys and girls in their ability to adhere to their diets, but should be more sensitized to this possibility in the case of female patients. Females also reported poorer adherence to diet than males and less confidence in their ability to overcome obstacles to dietary adherence. Clearly, gender differences in IBD adherence deserve further study.

There were some limitations to this study. First, the targeted population was specialized to a certain age group with a diagnosis of IBD living in an urban locale in the Southeastern United States, and the vast majority of the sample (84.1\%) was Caucasian and from the same general region. Furthermore, availability of participants was limited resulting in a small sample size, which resulted in inadequate power for some analyses. Given lack of power, the interaction 
analyses might be considered exploratory in nature and might have been statistically significant with a larger sample. However, when increasing sample size to heighten power, it is important to consider whether statistically significant findings are also clinically significant. Second, even though there was consistency between different measures of the same construct, the measures used were all self-report and thus factors such as subjectivity, method variance, and response bias need to be taken into account. Future work might use multi-method and multi-informant procedures in an effort to avoid these problems. The measures used for the current study were also modified from their original form and adapted for use with the current population. This was due to a lack of available measures specific to adolescents with IBD. These modifications might have affected the validity of the scales used, and for this reason total scores were not independently interpreted or compared to norms available in the literature. Third, the difficulties of assessing adherence have been discussed extensively in the literature and include problems with operationally defining adherence and accurate measurement (La Greca \& Bearman, 2003; Manne, 1998; Rapoff, 1999). The use of daily logs for the purpose of recording medication taking and diet is considered more accurate than retrospective reporting due to shorter recall periods (La Greca \& Bearman, 2003). In regard to the diet log, for the sake of parsimony and quantification of the data, daily logs were worded so that it was left up to the participants to decide whether or not their daily food intake was consumed according to medical advice. Thus, the assumption was made that the patients had accurate information on what foods are best avoided, either from their healthcare providers or their own experience.

Although this study was conducted with a small sample and has limitations, the current findings are provocative and suggest additional exploration. Therefore, it is suggested that future efforts are divided between three major areas: (a) further research on the role of diet and body 
satisfaction in IBD, (b) incorporation of developmental factors and gender differences into the exploration of predictors of medication and diet adherence, and (c) physician and patient education and clinical interventions to increase patient adherence.

IBD is an illness in which nutrition and diet play a significant role, especially in adolescents, due to the increased nutritional needs and potential for growth delays. Furthermore, diet adherence is a central part of their healthcare regimen in patients for who diet supplements or TPN are mandated. However, we currently do not have a good understanding of ways to incorporate diet into the treatment regimen and to prevent malnutrition, although efforts in this direction are being made (Wild et al., 2007). Beyond IBD, diet adherence is significant for the progress of many chronic illnesses, and can compromise optimal medical outcome. Adherence should not be viewed as a static characteristic of the patient's behavior, as it changes across the lifetime with the influence of various developmental factors. Age and body satisfaction appear to be part of this equation, but others should be explored including that of gender. These results are important, both for the purpose of informing patients, and also for more accurate differential diagnosis. IBD has been reported to be complicated in its presentation, diagnosis, and treatment, by an eating disorder due to the presence of fear of obesity, weight loss, and body image disturbances (for a review, see Powers, 1997).

Finally, it is critical that healthcare providers and patients gain a good understanding of the knowledge available on both medical treatments and diet. In IBD, patient education has been found to increase patient satisfaction, whereas a trend for increasing medication adherence has also been found (Waters, Jensen, \& Fedorak, 2005). Therefore, in light of the current findings, alerting families to the relation of age and body satisfaction, and diet adherence could increase their ability to work towards adherence. Research on potential interventions such as increasing 
the patient's control over weight management or the benefit of exercise would provide practical guidelines to optimize medical outcome through increased dietary adherence. 


\section{REFERENCES}

Abbott, J., Conway, S., Etherington, C., Fitzjohn, J., Gee, L., Morton, A., Musson, H., \& Webb, A.K. (2000). Perceived body image and eating behavior in young adults with cystic fibrosis and their healthy peers. Journal of Behavioral Medicine, 23, 501-517.

Aiken, L.S., \& West, S.G. (1991). Multiple regression: Testing and interpreting interactions. Sage Publications, Inc.

American Psychiatric Association. (2000). Diagnostic and statistical manual of mental disorders ( $4^{\text {th }}$ ed. - text revision). Washington, DC: Author.

Antisdel, J.E., \& Chrisler, J.C. (2000). Comparison of eating attitudes and behaviors among adolescent and young women with type 1 diabetes mellitus and phenylketonuria. Developmental and Behavioral Pediatrics, 21, 81-86.

Booth, I.W. (1991). The nutritional consequences of gastrointestinal disease in adolescence. Acta Paediatrica Scandinavica, Suppl. 373, 91-102.

Casati, J., Toner, B.B., de Rooy, E., Drossman, D.D., \& Maunder, R.G. (2000). Concerns of patients with inflammatory bowel disease. Digestive Diseases and Sciences, 45, 26-31.

Crohn's and Colitis Foundation of America (2005). Living with Crohn's disease. [Brochure]. NY: Author.

De Rooy, E.C., Toner, B.B., Maunder, R.G., Greenberg, G.R., Baron, D., Steinhart, H., et. al. . (2001). Concerns of patients with inflammatory bowel disease: Results from a clinical population. The American Journal of Gastroenterology, 96, 1816-1821.

Dunker, M.S., Stiggelbout, A.M., van Hogenzand, R.A., Ringers, J., Griffioen, G., \& Bemelman, W.A. (1998). Cosmesis and body image after laparoscopic-assisted and open ileocolic esection for Crohn's disease. Surgical Endoscopy, 12, 1334-1340. 
Escher, J.C., \& Taminiau, J.A.J.M. (2001). Treatment of inflammatory bowel disease in childhood. Scandinavian Journal of Gastroenterology, 36, Suppl. 234, 48-50.

Joyce, T.A. (2002). Body image and treatment adherence in adolescents and youth adults with cancer. (Doctoral dissertation, A \& M University, 2002). Dissertation Abstracts International, 62(7-B), 3380.

Kane, S.V. (2006). Systematic review: Adherence issues in the treatment of ulcerative colitis. Alimentary Pharmacology and Therapeutics, 23, 577-585.

Kelsay, K., Hazel, N.A., \& Wamboldt, M.Z. (2005). Predictors of body dissatisfaction in boys and girls with asthma. Journal of Pediatric Psychology, 30, 522-531.

Kraemer, H. C., Kazdin, A. E., Offord, D. R., Kessler, R. C., Jensen, P. S., \& Kupfer, D. J. (1999). Measuring the potency of risk factors for clinical or policy significance. Psychological Methods, 4, 257-271.

La Greca, A.M., \& Bearman, K.J. (2003). Adherence to pediatric treatment regimens. In M.C. Roberts (Ed.) Handbook of pediatric psychology. NY: The Guilford Press.

Lokken, K, Ferraro, F.R., Kirchner, T., \& Bowling, M. (2003). Gender differences in body size dissatisfaction among individuals with low, medium, or high levels of body focus. Journal of General Psychology, 130, 305-310.

López-Sanromán, A., \& Bermejo, F. (2006). Review article: How to control and improve adherence to therapy in inflammatory bowel disease. Alimentary Pharmacology and Therapeutics, 24 (suppl.3), 45-49.

MacDermott, R.P. (2007). Treatment of irritable bowel syndrome in outpatients with inflammatory bowel disease using a food and beverage intolerance, food and beverage avoidance diet. Inflammatory Bowel Disease 13, 91-96. 
Mackner, L.M., McGrath, A.M., \& Stark, L.J. (2001). Dietary recommendations to prevent and manage chronic pediatric health conditions: Adherence, interventions, and future directions. Developmental and Behavioral Pediatrics, 22, 130-143.

Manne, S.L. (1998). Treatment adherence and compliance. In R.T. Ammerman \& J.V. Campo (Eds.) Handbook of pediatric psychology and psychiatry: Disease, injury, illness (vol. II). Boston: Allyn \& Bacon.

National Center for Health Statistics and National Center for Chronic Disease Prevention and Health Promotion. (2000). CDC Growth Charts: United States: Body-mass-index-for-age percentiles: Boys, 2 to 20 years old. Atlanta: Center for Disease Control.

National Center for Health Statistics and National Center for Chronic Disease Prevention and Health Promotion. (2000). CDC Growth Charts: United States: Body-mass-index-for-age percentiles: Girls, 2 to 20 years old. Atlanta: Center for Disease Control.

Pharres, V., Steinberg, A.R., \& Thompson, J.K. (2004). Gender differences in peer and parental influences: Body image disturbance, self-worth and psychological functioning in preadolescent children. Journal of Youth and Adolescence, 33, 421-429.

Pope, H.G., Phillips, K.A., \& Olivardia, R. (2000). The Adonis Complex. The Free Press, New York.

Powers, P.S. (1997). Management of patients with comorbid medical conditions. In D.M. Garner \& P.E. Garfinkel (Eds.) Handbook of treatment for eating disorder. $2^{\text {nd }}$ ed. New York City, NY: The Guilford Press.

Rapoff, M.A. (1999). Adherence to pediatric medical regimens. New York: Kluwer Academic/ Plenum Publishers. 
Schlundt, D.G., Rea, M., Hodge, M., Flannery, M.E., Kline, S., Meek, J., Kinzer, C., \& Pichert, J.W. (1996). Assessing and overcoming situational obstacles to dietary adherence in adolescents with IDDM. Journal of Adolescent Health, 19, 282-288.

Stark, L.J. (2000). Adherence to diet in chronic conditions: The example of cystic fibrosis. In D. Drotar (Ed.) Promoting adherence to medical treatment in chronic childhood illness: concepts, methods, and interventions. Mahwah, NJ: Lawrence Erlbaum.

Veron-Guidry, S., \& Williamson, D.A. (1996). Development of a body image assessment procedure for children and preadolescents. International Journal of Eating Disorders, 20, 287-293.

Waters, B.M., Jensen, L., \& Fedorak, R.N. (2005) Effects of formal education for patients with inflammatory bowel disease: A randomized controlled trial. Canadian Journal of Gastroenterology, 19, 235-244.

Walters, S. (2001). Sex differences in weight perception and nutritional behaviour in adults with cystic fibrosis. Journal of Human Nutrition and Dietetics, 14, 83-91.

Wild, G.E, Drozdowski, L, \& Tartaglia, C. (2007). Nutritional modulation of the inflammatory response in inflammatory bowel disease - From the molecular, to the integrative, to the clinical. World Journal of Gastroenterology, 13, 1-7.

Williamson, D.A., Davis, C. J., Bennett, S. M., Gorecrny, A.J., \& Gleaves, D.H. (1989). Development of a simple procedure for body image assessment. Behavioral Assessment, 11, 433-446.

Woolner, J.T., Parker, T.J., Kirby, G.A., \& Hunter, J.O. (1998).The development and evaluation of a diet for maintaining remission in Crohn's disease. Journal of Human Nutrition and Diet, 11, 1-11. 


\section{ACKNOWLEDGEMENTS}

This study was supported by a Georgia State University dissertation grant awarded to the first author under the direction of the second. This study was presented at the annual meeting of the Society for Behavioral Medicine, 2007. The authors thank Don Bearden, Kara Leffingwell, and Latrice Lewis, for their assistance with the data collection process. 
Table 1

Descriptive statistics for study variables

\begin{tabular}{|c|c|c|c|c|c|}
\hline Variable & $N$ & $M$ & $S D$ & MIN & $M A X$ \\
\hline \multicolumn{6}{|l|}{ Predictor Variables } \\
\hline Age & 44 & 16.64 & 2.42 & 10 & 21 \\
\hline \multicolumn{6}{|l|}{ Body satisfaction } \\
\hline \multicolumn{6}{|l|}{$\mathrm{BSQ}^{1}$ - Total Score } \\
\hline Male & 22 & 36.45 & 4.88 & 26 & 45 \\
\hline Female & 22 & 33.52 & 7.77 & 10 & 45 \\
\hline \multicolumn{6}{|c|}{ BIA $^{2}$ - Body Dysphoria Score } \\
\hline Male & 22 & .41 & .85 & -1 & 3 \\
\hline Female & 22 & .77 & .92 & -1 & 3 \\
\hline
\end{tabular}

Dependent variables

Diet adherence

$\begin{array}{lccccc}\text { SODA }^{3} & 43 & 417.74 & 63.21 & 268 & 543 \\ \text { Diet Log } & 40 & 18.23 & 2.33 & 14 & 21 \\ \text { Diet Adherence Perception } & 42 & 60.48 & 28.03 & 0 & 100\end{array}$

Note. ${ }^{1}$ Body Satisfaction Questionnaire; ${ }^{2}$ Body Image Assessment; ${ }^{3}$ Situational Obstacles for Dietary Adherence. 
Table 2

Regression analyses examining main effects and interactions among age and body satisfaction in predicting dietary adherence.

\begin{tabular}{|c|c|c|c|c|c|c|c|c|c|c|c|c|}
\hline & \multicolumn{6}{|c|}{ Body Satisfaction Measured by the BIA ${ }^{I}$} & \multicolumn{6}{|c|}{ Body Satisfaction Measured by the $B S Q^{2}$} \\
\hline & \multicolumn{2}{|c|}{$\mathrm{SODA}^{3}$} & \multicolumn{2}{|c|}{ Diet Log } & \multicolumn{2}{|c|}{ D Adh Perc $^{4}$} & \multicolumn{2}{|c|}{$\mathrm{SODA}^{3}$} & \multicolumn{2}{|c|}{ Diet Log } & \multicolumn{2}{|c|}{ D Adh Perc ${ }^{4}$} \\
\hline & $\beta$ & $\Delta \mathrm{R}^{2}$ & $\beta$ & $\Delta \mathrm{R}^{2}$ & $\beta$ & $\Delta \mathrm{R}^{2}$ & $\beta$ & $\Delta \mathrm{R}^{2}$ & $\beta$ & $\Delta \mathrm{R}^{2}$ & $\beta$ & $\Delta \mathrm{R}^{2}$ \\
\hline Step 1: & & $.17 * * *$ & & .01 & & $.07 *$ & & $.17 * * *$ & & .01 & & $.07 *$ \\
\hline Age & -.42 & & -.09 & & -.26 & & -.42 & & -.09 & & -.26 & \\
\hline Step 2: & & $.1^{*}$ & & $.28 * * *$ & & $.1 *$ & & $.12 * *$ & & $.23 * * *$ & & .06 \\
\hline Body Satisfaction & -.33 & & -.31 & & -.37 & & .36 & & .19 & & -.27 & \\
\hline Step 3: & & .03 & & .05 & & .06 & & .07 & & .04 & & .04 \\
\hline Body Satisfaction x Gender & -.12 & & -.08 & & -.15 & & .28 & & -.15 & & .01 & \\
\hline Body Satisfaction x Age & .13 & & .05 & & -.05 & & .08 & & -.01 & & .11 & \\
\hline Gender x Age & -.05 & & -.22 & & -.22 & & .04 & & -.2 & & -.11 & \\
\hline Step 4: & & .04 & & .00 & & .01 & & .05 & & .01 & & .00 \\
\hline Body Satisfaction x Gender x Age & -.24 & & .01 & & -.15 & & -.28 & & -.18 & & -.05 & \\
\hline
\end{tabular}

Note. ${ }^{1}$ Body Image Assessment; ${ }^{2}$ Body Satisfaction Questionnaire; ${ }^{3}$ Situational Obstacles to Dietary Adherence; ${ }^{4}$ Diet Adherence Perception $* p<.10, * * p<.05, * * * p<.01$ 